Schmerz 2022 $\cdot 36: 172-181$

https://doi.org/10.1007/s00482-021-00590-1

Angenommen: 16. August 2021

Online publiziert: 7. Oktober 2021

๑) Der/die Autor(en) 2021

\section{Ergebnisse einer Pilotstudie zur Rolle der Therapieerwartung bei der interdisziplinären multimodalen Schmerztherapie bei chronischem Rückenschmerz}

\author{
Dustin Maser · Daniel Müller · Ulrike Bingel · Diana Müßgens \\ Klinik für Neurologie, Zentrum für Translationale Neuro- und Verhaltenswissenschaften und Zentrum für \\ universitäre Schmerzmedizin, Universitätsklinikum Essen (AöR), Essen, Deutschland
}

Hintergrund: Chronische Rückenschmerzen sind eine schwerwiegende und global sehr häufig auftretende Erkrankung mit enormen persönlichen sowie sozioökonomischen Auswirkungen. Die interdisziplinäre multimodale Schmerztherapie (IMST) ist eines der wenigen evidenzbasierten Behandlungsverfahren für chronische Schmerzen. Obwohl bekannt ist, dass Schmerzen sowie deren Chronifizierung und Behandlung von den persönlichen Erwartungen der Patienten beeinflusst werden, gibt es wenige etablierte Interventionen oder Richtlinien für eine aktive Modulation dieses Effekts.

Ziel der Arbeit: Wir möchten mit dieser Arbeit die Rolle der Erwartung als Prädiktor für Schmerzen sowie schmerzbezogene Beeinträchtigung in der klinischen Praxis verdeutlichen und präsentieren hierzu beispielhaft explorative Pilotdaten einer Beobachtungskohorte unserer Klinik.

Material und Methoden: Die Untersuchung zeigt erste Daten einer prospektiven longitudinalen Beobachtungsstudie bestehend aus bis zu 41 Patienten mit chronischen Rückenschmerzen, die im Setting einer IMST am Essener Rückenschmerz-Zentrum behandelt wurden. Es wurden Daten zum Zeitpunkt der Aufnahme (T0) und der Entlassung (T1) sowie drei Monate nach Therapieende (T2) erhoben. Primäre Endpunkte waren die Schmerzintensität und die Schmerzbeeinträchtigung. Zusätzlich erfassten wir die Therapieerwartung zum Zeitpunkt der Aufnahme als möglichen Prädiktor. Die Bedeutung der vor der Therapie erhobenen Therapieerwartung wurde mittels linearer Regression erfasst.

Ergebnisse: Die IMST führte zu einer signifikanten Besserung in Bezug auf die Schmerzintensität und -beeinträchtigung. Der Effekt auf die Schmerzintensität war über den Zeitraum von drei Monaten nach Therapieende anhaltend und die Beeinträchtigung sank in diesem Zeitraum weiter signifikant.

Diskussion: Erwartung war ein signifikanter Prädiktor für die Abnahme der Schmerzintensität und erklärte ca. 15\% der Varianz. In der klinischen Praxis sollten daher valide Methoden etabliert werden, negative Erwartungen zu reduzieren und positive Erwartungen zu fördern.

Wir bestätigen hiermit, dass es sich bei dem Manuskript um eine Originalarbeit handelt und sie nicht zum Review bei einer anderen Fachzeitschrift vorliegt.

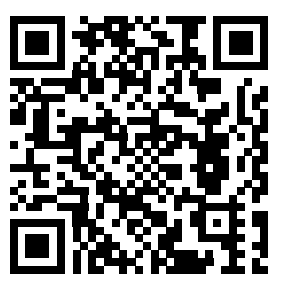

\section{Schlüsselwörter}

Chronischer Rückenschmerz • Interdisziplinäre multimodale Schmerztherapie (IMST) ·

Therapieerwartung · Placebo · Longitudinale Beobachtungsstudie 


\section{Hintergrund und Fragestellung}

\section{Chronische Rückenschmerzen}

Rückenschmerz gilt weltweit als eine der Hauptursachen für Aktivitätseinschränkungen und Arbeitsausfälle [26], was zu einer hohen sozioökonomischen und individuellen Belastung führt [17]. Etwa $90 \%$ aller Rückenschmerzen sind unspezifisch und somit nicht zurückführbar auf einen einfachen pathoanatomischen Zusammenhang [39]. Persistieren Rückenschmerzen länger als drei bis sechs Monate gelten sie als chronisch [62]. Obwohl lediglich ca. 10-15\% aller Fälle chronifizieren [67], scheinen gerade diese Fälle hochgradig therapieresistent zu sein [21].

\section{IMST}

Die interdisziplinäre multimodale Schmerztherapie (IMST) ist ein evidenzbasiertes Therapieverfahren bei chronischen Schmerzen [52] und gilt in Deutschland als Goldstandard zur Behandlung chronischer Schmerzen [27]. Viele nationale und internationale Studien bestätigen die Effektivität der IMST für unterschiedliche chronische Schmerzsyndrome [20, 23, 29, 57]. Dennoch zeigen einige Übersichtsartikel nur eine schwache oder moderate Evidenz und unterstreichen die Notwendigkeit qualitativ hochwertigerer Studien $[16,29,45]$. So gibt es im internationalen Raum immer noch keine einheitliche Definition für IMST, und evaluierte Therapien unterscheiden sich dadurch oft in Inhalt und Qualität [28]. Darüber hinaus ist unklar welche, der vielen Inhalte wirksam sind und wie sie mit Patientenvariablen interagieren. Erste Schritte wurden in Deutschland getätigt, indem detaillierte Konzepte für Struktur-, Prozess- und Ergebnisparameter erarbeitet wurden [1, 2, 53]. Im internationalen Vergleich bietet die deutsche Schmerztherapielandschaft somit einen besonderen und chancenreichen Kontext, um die Effekte von Patientenvariablen unter vergleichsweise standardisierten Bedingungen zu untersuchen und diesbezügliche Interventionen zu entwickeln. Insbesondere die Therapierwartung scheint hier eine wichtige Rolle zu spielen.
Die Rolle der Erwartung bei Schmerzen

Eine zunehmende Zahl internationaler Studien unterstreicht den Einfluss von Erwartung auf den Schmerz und seine Behandlung [24, 42, 49]. Die Erwartung gilt als eines der Kernelemente des Placebo- sowie Noceboeffekts [3] und kann somit einen positiven oder negativen Einfluss auf die Behandlung und den Krankheitsverlauf haben. Dies gilt in Anwesenheit eines bekannten Wirkfaktors, beispielsweise eines Medikaments, aber auch in seiner Abwesenheit. Therapieerwartungen können sowohl Schmerzen als auch die Wirkung einer analgetischen Behandlung verstärken oder abschwächen [8]. Dies lässt sich durch subjektive Patientenaussagen und durch Messungen der neuronalen Aktivität in den schmerzbezogenen Kortizes belegen [31]. Für Details siehe Asan et al. in diesem Themenheft.

Präoperative Erwartungen beeinflussen schmerzbezogene Ergebnisse bei unterschiedlichen chirurgischen Eingriffen [4], und sogar Scheinoperationen können zu einer Symptomlinderung führen [41, 44]. Erwartungen beeinflussen ebenfalls die Rehabilitation nach akuten Körpertraumata [46]. Positive Erwartungen gelten als protektiver Faktor im Übergang von akuten zu chronischen Schmerzen [42]. Auch bei chronischen Schmerzen ist der modulierende Einfluss von positiven Erwartungen belegt $[14,22,70]$. So unterstützen Erwartungen die Ergebnisse von Akupunktur [70], kognitiver Verhaltenstherapie [22] und multimodaler Schmerztherapie bei chronischen Schmerzen [14].

Auch negative Erwartungen beeinflussen das Therapieergebnis und sind ein häufiges Phänomen im klinischen Alltag [47]. So entwickeln Patienten nach entsprechender Aufklärung auch Nebenwirkungen, die nicht zum bekannten Nebenwirkungsspektrum dieses Medikaments gehören [8, 19]. Aversive Reize können ebenfalls verstärkt werden. Der Hinweis, ein Nadelstich könnte schmerzen, führt so zu einer Hyperalgesie [65]. Gleichsam können negative Erwartungen, Sorgen und Ängste bezüglich einer Behandlung unerwünschte Nebenwirkungen von medikamentösen Behandlungen verstärken [36, 38]. Die Erwartungshal- tung ist also ein bedeutsamer Prädiktor für die Schmerztherapie, da sie das Therapieergebnis sowohl positiv als auch negativ beeinflussen kann.

\section{Ziele der Pilotstudie}

Ziel dieser Pilotstudie war es, den Einfluss der Therapieerwartung auf das Ergebnis einer interdisziplinären multimodalen Schmerztherapie bei chronischen Rückenschmerzen zu untersuchen. Die Ergebnisse zielen darauf $a b$, auf das Ausmaß dieser Einflussgröße hinzuweisen. Darüber hinaus könnten sie eine Grundlage bilden, um Therapieergebnisse durch gezielte erwartungsmodulierende Interventionen zu optimieren.

\section{Studiendesign und Unter- suchungsmethoden}

\section{Behandlung am Essener \\ Rückenschmerz-Zentrum}

Das Essener Rückenschmerz-Zentrum existiert seit 2015 und behandelt regelhaft 130 Patienten jährlich mit der IMST. Patienten werden zumeist regional aus der RheinRuhr-Region oder seltener auch überregional zu uns verwiesen.

Die IMST am Essener RückenschmerzZentrum entspricht den Mindestvorgaben des amtlichen Operationen- und Prozedurenschlüssels (OPS) [6] und orientiert sich zusätzlich an den Empfehlungen zu Struktur- und Prozessparametern der Adhoc-Kommission "Interdisziplinäre Multimodale Schmerztherapie" der Deutschen Schmerzgesellschaft e.V. [51]. Über die zwei geforderten Fachdisziplinen hinaus besteht das Behandlungsteam am Essener Rückenschmerz-Zentrum aus Medizinern unterschiedlicher Fachrichtungen, Physiotherapeuten und Psychotherapeuten, welche im stetigen interdisziplinären Austausch mit dem Patienten arbeiten. Die Behandlungstage zählen 14-21 Tage für stationäre und 10-15 Tage für teilstationäre Patienten und übersteigen damit die 7-Tage Vorgabe des OPS. Die Anwendungen beinhalten unter anderem medikamentöse Einstellung, Psychotherapie, Physiotherapie, Psycho-, Physiosowie medizinische Edukation und Trainingstherapie. Auch dies überschreitet die 
Tab. 1 Vollständige Datensets (T0-T2) und Erwartungsfragebogen

\begin{tabular}{|l|l|l|}
\hline & Schmerzintensität & Beeinträchtigung \\
\hline Alle Gruppen $(n[\%])$ & $37[100 \%]$ & $41[100 \%]$ \\
\hline Alter $(\mathrm{M} \pm$ SD [min-max]) & $54,78 \pm 15,20[29-83]$ & $55,46 \pm 14,95[29-83]$ \\
\hline $\begin{array}{l}\text { Arbeitsunfähigkeit }{ }^{\boldsymbol{a}}(\mathrm{M} \pm \mathrm{SD} \text { [min- } \\
\text { max]) }\end{array}$ & $47,32 \pm 37,27[0-92]$ & $45,10 \pm 36,14[0-92]$ \\
\hline
\end{tabular}

\section{Geschlecht}

\begin{tabular}{|c|c|c|}
\hline Weiblich $(n[\%])$ & $23[62,2 \%]$ & $26[63,4 \%]$ \\
\hline Männlich $(n[\%])$ & $14[37,8 \%]$ & $15[36,6 \%]$ \\
\hline \multicolumn{3}{|l|}{ Wohnsituation } \\
\hline Alleine ( $n[\%])$ & $9[24,3 \%]$ & $10[24,4 \%]$ \\
\hline In Partnerschaft ( $n$ [\%]) & $22[59,5 \%]$ & $24[58,5 \%]$ \\
\hline Anders ( $n[\%])$ & $0[0,0 \%]$ & $1[2,4 \%]$ \\
\hline NA $(n[\%])$ & $6[16,2 \%]$ & $6[14,6 \%]$ \\
\hline \multicolumn{3}{|l|}{ Medikamentenfehlgebrauch } \\
\hline $\mathrm{Ja}(n[\%])$ & $9[24,3 \%]$ & $9[22,0 \%]$ \\
\hline Nein $(n[\%])$ & $28[75,7 \%]$ & $32[78,0 \%]$ \\
\hline $\begin{array}{l}\text { Schmerzintensität TO }(\mathrm{M} \pm \mathrm{SD} \\
[\mathrm{min}-\mathrm{max}])\end{array}$ & $66,49 \pm 20,85[20,00-83,3]$ & $66,50 \pm 20,12[20,00-93,3]$ \\
\hline $\begin{array}{l}\text { Beeinträchtigung TO }(\mathrm{M} \pm \mathrm{SD}[\mathrm{min}- \\
\max ])\end{array}$ & $\begin{array}{l}70,45 \pm 22,96 \\
{[13,33-100,00]}\end{array}$ & $\begin{array}{l}71,54 \pm 22,01 \\
{[13,33-100,00]}\end{array}$ \\
\hline
\end{tabular}

\section{Grad der Chronifizierung (von Korff)}

\begin{tabular}{|c|c|c|}
\hline $1(n[\%])$ & $2[5,4 \%]$ & $2[4,9 \%]$ \\
\hline $2(n[\%])$ & $3[8,1 \%]$ & $3[7,3 \%]$ \\
\hline $3(n[\%])$ & $9[24,3 \%]$ & $9[22,0 \%]$ \\
\hline $4(n[\%])$ & $23[62,2 \%]$ & $27[65,9 \%]$ \\
\hline $\begin{array}{l}\text { Komorbiditäten }{ }^{b}(\mathrm{M} \pm \mathrm{SD} \text { [min- } \\
\text { max]) }\end{array}$ & $3,22 \pm 2,25[0-10]$ & $3,39 \pm 2,21[0-10]$ \\
\hline \multicolumn{3}{|l|}{ Psychische Auffälligkeiten (DASS) } \\
\hline Depression $(\mathrm{M} \pm \mathrm{SD}$ [min-max]) & $6,92 \pm 4,30[1-16]$ & $7,76 \pm 4,92[1-19]$ \\
\hline Klinisch auffällig ( $n[\%])$ & $8[21,6 \%]$ & $12[70,7 \%]$ \\
\hline Unauffällig ( $n$ [\%]) & $29[78,4 \%]$ & $29[29,3 \%]$ \\
\hline Angst $(\mathrm{M} \pm \mathrm{SD}[$ min-max]) & $4,32 \pm 4,83[0-19]$ & $4,66( \pm 4,76)[0-19]$ \\
\hline Klinisch auffällig ( $n$ [\%]) & $9[24,3 \%]$ & $12[70,7 \%]$ \\
\hline Unauffällig ( $n$ [\%]) & $28[75,7 \%]$ & $29[29,3 \%]$ \\
\hline Stress $(M \pm S D$ [min-max]) & $7,92 \pm 4,73[1-19]$ & $8,22 \pm 4,86[1-19]$ \\
\hline Klinisch auffällig ( $n[\%])$ & $13[35,1 \%]$ & $15[63,4 \%]$ \\
\hline Unauffällig ( $n$ [\%]) & $24[64,9 \%]$ & $26[36,6 \%]$ \\
\hline $\begin{array}{l}\text { Wohlbefinden (FW-7) }(\mathrm{M} \pm \mathrm{SD} \\
[\mathrm{min}-\mathrm{max}])\end{array}$ & $15,19 \pm 7,49[2-32]$ & $14,83 \pm 8,33[0-33]$ \\
\hline Beeinträchtigt $(n[\%])$ & $12[32,4 \%]$ & $14[34,1 \%]$ \\
\hline Normal ( $n[\%])$ & $25[67,6 \%]$ & $27[65,9 \%]$ \\
\hline
\end{tabular}

Mindestanforderung von drei übenden Verfahren. Die Therapie wird vorrangig im Gruppensetting durchgeführt, wobei täglich mehrere Gruppen stattfinden (je 60 bis $90 \mathrm{~min})$. Darüber hinaus erhält jeder Patient eine wöchentliche physiotherapeutische Einzelsitzung (je $30 \mathrm{~min}$ ) sowie psychotherapeutische (je $45 \mathrm{~min}$ ) und ärztliche Einzelgespräche (je $30 \mathrm{~min}$ ). Entspannungs- oder Achtsamkeitsübungen werden am Ende eines jeden Tages ange- leitet (je $30 \mathrm{~min}$ ). An den Wochenenden erhalten die Patienten psychotherapeutische Aufgaben und sollen die erlernten Bewältigungsstrategien in Eigenarbeit üben.

Die Patienten, die zur IMST erscheinen, leiden oftmals unter therapieresistenten chronischen Rückenschmerzen und sind in ihrer Arbeitsfähigkeit oder Lebensqualität stark eingeschränkt. Häufig berichten die Patienten von jahrelangen „Ärzte-
Odysseen", sowie unzähligen misslungenen unimodalen Therapieversuchen, die von ambulanter Medikation bis hin zu mehrfachen fehlgeschlagenen Wirbelsäulenoperationen reichen. Bei den meisten Patienten werden die Schmerzen durch eine Kombination verschiedener biopsychosozialer Faktoren aufrechterhalten, und sie erfüllen damit zumeist die Kriterien der Diagnose F45.41 chronische Schmerzstörung mit somatischen und psychologischen Faktoren des ICD-10 [5]. Darüber hinaus leiden diese Patienten oft unter somatischen und/oder psychischen Komorbiditäten sowie unter einem Medikamentenfehlgebrauch.

\section{Studiendesign}

Es wurden Patienten mit chronischen Rückenschmerzen im Alter von 29 bis 83 Jahren untersucht, die zwischen Januar 2019 und Februar 2020 am Essener Rückenschmerz-Zentrum im Rahmen einer IMST behandelt wurden. Die Indikation zur IMST wurde nach den Vorgaben des amtlichen Operationen- und Prozedurenschlüssels (OPS) [6] sowie den Empfehlungen der Ad-hoc-Kommission „Multimodale interdisziplinäre Schmerztherapie" [12], gestellt. Bei entsprechender Indikation wurden Patienten zu einer zweioder dreiwöchigen IMST eingeladen, die im stationären oder teilstationären Setting erfolgen konnte. Die Auswahl des Settings wurde vorrangig von den Versicherungskonditionen und, in geringerem Maße, auch von den medizinischen Komorbiditäten der Patienten bestimmt. Eine Therapiegruppe bestand im Regelfall aus 6-7 stationären und 1-2 teilstationären Patienten. Ob eine zwei- oder dreiwöchige IMST durchgeführt wurde, hing vorrangig von strukturellen Variablen wie der Länge der Warteliste ab. Die Therapieinhalte waren jedoch unabhängig von Dauer und Setting. Die Patienten füllten den angepassten deutschen Schmerzfragebogen [50] im Rahmen des standardisierten Therapie-Monitorings zu vier verschiedenen Messzeitpunkten aus: Zur Aufnahme (T0), zur Entlassung (T1), sowie drei (T2), sechs (T3) und zwölf Monate (T4) nach der Therapie. Für die vorliegende Pilotstudie wurden ausschließlich die ersten drei Messzeitpunkte (T0-T2) inkludiert, da 


\begin{tabular}{|c|c|c|}
\hline & Schmerzintensität & Beeinträchtigung \\
\hline \multicolumn{3}{|l|}{ Lebensqualität (SF12) } \\
\hline $\begin{array}{l}\text { Körperl. Gesundheit (M } \pm \text { SD [min- } \\
\text { max]) }\end{array}$ & $29,17 \pm 9,89[8,41-52,14]$ & $29,13 \pm 9,58[8,41-52,14]$ \\
\hline $1 \times S D$ niedriger $^{c}(n[\%])^{c}$ & $8[21,6 \%]$ & $9[22,0 \%]$ \\
\hline Norm Schmerzpatient ${ }^{\complement}(n[\%])$ & $24[64,9 \%]$ & $27[65,9 \%]$ \\
\hline $1 \times S D$ höher $(n[\%])$ & $5[13,5 \%]$ & $5[12,2 \%]$ \\
\hline $\begin{array}{l}\text { Geist. Gesundheit }{ }^{\complement}(M \pm S D \text { [min- } \\
\text { max]) }\end{array}$ & $\begin{array}{l}39,42 \pm 13,35 \\
{[17,21-66,27]}\end{array}$ & $\begin{array}{l}38,33 \pm 13,13 \\
{[17,21-66,27]}\end{array}$ \\
\hline $1 \times S D$ niedriger $(n[\%])$ & $18[48,6 \%]$ & $22[53,7 \%]$ \\
\hline Norm Schmerzpatient ${ }^{\complement}(n[\%])$ & $12[32,4 \%]$ & $12[29,3 \%]$ \\
\hline $1 \times S D$ höher $(n[\%])$ & $7[18,9 \%]$ & $7[17,1 \%]$ \\
\hline \multicolumn{3}{|l|}{ Setting } \\
\hline Stationär ( $n[\%])$ & $30[81,1 \%]$ & $34[82,9 \%]$ \\
\hline Teilstationär $(n[\%])$ & $7[18,9 \%]$ & $7[17,1 \%]$ \\
\hline \multicolumn{3}{|l|}{ Dauer } \\
\hline 2-Wochen $(n[\%])$ & $9[24,3 \%]$ & $11[26,8 \%]$ \\
\hline 3-Wochen $(n[\%])$ & $28[75,7 \%]$ & $30[73,2 \%]$ \\
\hline \multicolumn{3}{|c|}{$\begin{array}{l}\text { Arbeitsunfähigkeit gemessen anhand Anzahl Tage, an denen in den letzten drei Monaten Arbeitsun- } \\
\text { fähigkeit vorlag } \\
\text { bKomorbiditäten wurden anhand der Diagnosen in den jeweiligen Arztbriefen erfasst und gezählt. So- } \\
\text { matische Korrelate der Rückenschmerzen sowie Verdachtsdiagnosen wurden hierbei ausgeschlossen } \\
\text { 'Bezieht sich auf die Validierungsstichprobe 2006, mit Mittelwerten und Standardabweichungen, aus } \\
\text { dem Handbuch zum Deutschen Schmerzfragebogen [50] }\end{array}$} \\
\hline
\end{tabular}

zum Zeitpunkt der Datensichtung noch nicht ausreichende Datenmengen für die anderen Messzeitpunkte erschlossen wurden. Nur Patienten mit vollständigem Datensatz und ausgefülltem Erwartungsfragebogen wurden eingeschlossen.

Grundsätzlich wurden alle Datenerhebungen online über die verschlüsselte Plattform PainPool ${ }^{\circledR}$ (smart-Q Softwaresysteme $\mathrm{GmbH}$ ) erhoben. Patienten erhielten maximal einen Monat im Voraus einen Link zur Internetplattform. Falls Patienten keine E-Mail-Adresse besaßen oder den Fragebogen nicht rechtzeitig ausgefüllt haben, wurden die Daten handschriftlich am Tag der Aufnahme, noch bevor die eigentliche Therapie begann, erhoben. Zur Entlassung wurden die Daten am Ende des letzten Therapietags mit Tablets erhoben. In Ausnahmefällen, wenn Patienten z.B. technisch nicht mit dem Tablet zurechtkamen, wurde die Messung handschriftlich erhoben. Die Daten der Eingangserhebung zählten dann als auswertbar, wenn sie frühestens einen Monat vor der Therapie und spätestens am Aufnahmetag ausgefüllt wurden. Die Daten der Entlass-Erhebung galten dann als auswertbar, wenn sie frühestens am
Patienten haben den Schmerzfragebogen zu T2 nicht beendet. Somit haben wir 37 vollständige schmerzbezogene und 41 vollständige beeinträchtigungsbezogene Datensätze gesammelt. Es ergibt sich ein Drop-out von 47 bzw. 51 (53,41\% bzw. 57,95\%). Zum Zeitpunkt T0 gibt es keine Unterschiede zwischen inkludierten Patienten und Drop-outs hinsichtlich der primären Endpunkte oder des Alters.

- Tab. 1 zeigt die Verteilung der Patienten auf die primären Endpunkte hinsichtlich Alter, Arbeitsfähigkeit, Geschlecht, Wohnsituation, Medikamentenfehlgebrauch, primäre Endpunkte zu T0, Grad der Chronifizierung nach von Korff [68], Komorbiditäten, psychologischen Auffälligkeiten nach der Depression, Anxiety and Stress Scale (DASS) [48], Beeinträchtigung des habituellen Wohlbefindens (FW-7) [25], der gesundheitsbezogenen Lebensqualität (körperlich, geistig) nach der deutschen Version des Veterans RAND 12 (SF12) [30], Behandlungsdauer und -setting. Abgesehen von einem Patienten mit einem "failed back surgery syndrome" (M96.1) trugen alle Patienten die Hauptdiagnose F45.41 - Chronische Schmerzstörung mit somatischen und psychischen Faktoren - ein. Unsere Stichprobe scheint hinsichtlich der erhobenen Variablen ungefähr der Normgruppe im „Handbuch Deutscher Schmerzfragebogen" zu entsprechen [50]. Lediglich die geistige Gesundheit scheint etwas niedriger als in der dort genannten Normgruppe von Schmerzpatienten.

\section{Fragebögen}

Die Schmerzintensität sowie die schmerzbedingte Beeinträchtigung wurden mit dem Fragebogen zur Graduierung chronischer Schmerzen nach von Korff erhoben [68]. Hierbei wird ein zusammengefasster Schmerzwert als Mittelwert aus der aktuellen, maximalen und durchschnittlichen Schmerzbewertung über die letzten vier Wochen, auf einer elf Punkte umfassenden numerischen Ratingskala (NRS) [0-10, Anker: „kein Schmerz" -, unerträglicher Schmerz"], berechnet und mit 10 multipliziert. Für die schmerzbedingte Beeinträchtigung wird der Mittelwert aus der subjektiven Beeinträchtigung im Alltag, bei Freizeitaktivitäten und bei der Arbeit, 
Tab. 2 Deskriptive Statistik

\begin{tabular}{|c|c|c|c|c|c|c|c|c|}
\hline & \multicolumn{2}{|c|}{ Aufnahme (T0) } & \multicolumn{2}{|c|}{ Entlassung (T1) } & \multicolumn{2}{|c|}{ 3-Monate nach Entlassung (T2) } & \multicolumn{2}{|c|}{ Linderung über alle Zeitpunkte } \\
\hline & $\mathrm{M} \pm \mathrm{SD}$ & Range & $M \pm S D$ & Range & $M \pm S D$ & Range & $M \pm S D$ & Range \\
\hline Schmerzintensität & $66,5 \pm 20,8$ & $20,0-93,3$ & $48,2 \pm 16,4$ & $10,0-76,7$ & $43,2 \pm 25,7$ & $0,0-93,3$ & $11,6 \pm 13,6$ & $-11,7-41,7$ \\
\hline Beeinträchtigung & $71,5 \pm 22,1$ & $13,3-100,0$ & $60,2 \pm 25,2$ & $0,0-100,0$ & $38,0 \pm 25,9$ & $0,0-100,0$ & $16,8 \pm 14,6$ & $5,0-50,0$ \\
\hline Erwartung $^{a}$ & $0,5 \pm 2,6$ & $-5,0-5,2$ & - & - & - & - & - & - \\
\hline Erwartung $^{b}$ & $0,3 \pm 2,6$ & $-5,0-5,2$ & - & - & - & - & - & - \\
\hline
\end{tabular}

bezogen auf die letzten drei Monate, auf einer elf Punkte umfassenden NRS [0-10, Anker: "keine Beeinträchtigung"„völlige Beeinträchtigung"], berechnet und ebenfalls mit 10 multipliziert.

Der in dieser Studie untersuchte mögliche Prädiktor für den Therapierfolg ist die Therapieerwartung. Diese wurde mit einer ins Deutsche übersetzten und für das Setting angepassten Version des Credibility/Expectancies Questionnaire (CEQ) nach Devilly und Borkovec gemessen [15]. Der Erwartungswert setzt sich aus drei Werten zusammen. Die erste Frage bezieht sich auf die Gedanken des Patienten und erhebt auf einer elf Punkte umfassenden NRS [0-100\%] den Prozentsatz der Linderung, welchen die Patienten durch die Therapie erwarten. Die zweite Frage bezieht sich auf das Gefühl und fragt auf einer drei Punkte umfassenden Likert Skala [„,̈̈berhaupt nicht", "etwas", "sehr"], wie sehr die Patienten wirklich fühlen, dass die Therapie helfen wird, ihre Symptome zu lindern. Die letzte Frage bezieht sich ebenfalls auf das Gefühl der Patienten und fragt auf einer elf Punkte umfassenden NRS [0-100\%] wie stark die Linderung, dem Gefühl der Patienten nach, tatsächlich nach der Therapie sein wird. Die Erwartungswerte werden in standardisierte Z-Scores umgewandelt und als Summe verrechnet. Je höher der Z-Score, desto größer ist auch die erwartete Linderung.

\section{Statistische Auswertung}

Zunächst wurden Schmerzintensität und schmerzbedingte Beeinträchtigung sowie die Erwartungswerte mittels deskriptiver Statistik analysiert. Um die Vorannahmen der Normalverteilung zu überprüfen, wurden Shapiro-Wilk-Tests durchgeführt sowie QQ-Plots und Dichteplots beurteilt.
Zur Überprüfung des Therapieeffekts wurden einfaktorielle ANOVAS mit Messwiederholungen für Schmerzintensität und Beeinträchtigung durchgeführt. Signifikante Veränderungen wurden anschließend durch Post-hoc-Paarvergleiche mit Bonferroni-Korrektur genauer definiert. Als Effektstärken wurden der generalisierte Eta-Quadrat für alle Messzeitpunkte sowie Cohens Delta für die Post-hoc-tTests bestimmt. Für die Prädiktorenanalyse wurden Änderungswerte für die primären Endpunkte berechnet. Hierzu wurde die für jeden Patienten die Steigung über alle drei Messzeitpunkte berechnet. Die resultierten Werte wurden mit -1 multipliziert. Somit gibt ein positiverer Wert eine größere Linderung an. Diese Linderungswerte wurden zunächst hinsichtlich ihrer Normalverteilung untersucht. Anschließend wurden lineare Regressionsanalysen mit jeweils der Schmerzlinderung sowie der Beeinträchtigungslinderung als abhängige und der Therapieerwartung als unabhängige Variable durchgeführt.

\section{Ergebnisse}

\section{Deskriptive Statistik}

Die Mittelwerte (M), Standardabweichungen (SD) sowie die Minima und Maxima der Schmerzintensität, der Beeinträchtigung und der Erwartung sind in - Tab. 2 aufgeführt. Einstichproben-t-Tests zeigen, dass die Linderung der Schmerzintensität und die der Beeinträchtigung jeweils größer als 0 sind (Schmerzlinderung: $\mathrm{t}(36)=5,3, p<0,0001, \mathrm{~d}=0,88$, großer Effekt; Beeinträchtigungslinderung $\mathrm{t}(40)=7,4, p<0,0001, \mathrm{~d}=1,15$, großer Effekt). Da die Schmerzintensität sowie die Beeinträchtigung zu T0 und die Beeinträchtigungslinderung eine leichte
Abweichung von der Normalverteilung zeigten, haben wir, wie von Tabachnick und Fidell [61] empfohlen, bei Linksschiefe eine invertierte Wurzeltransformation $\sqrt{(\max (x)+1)-x})$ angewandt. Bei Rechtsschiefe haben wir eine einfache Wurzeltransformation benutzt. Da die Transformationen zu den gleichen signifikanten Effekten führen, rapportieren wir zugunsten der Interpretierbarkeit der Skalen die Analysen der nichttransformierten Werte.

\section{Verlauf der Schmerzintensität und der Schmerzbeeinträchtigung}

Eine einfache ANOVA mit Messwiederholungen zeigte einen signifikanten Haupteffekt von Messzeitpunkt auf die Schmerzintensität $(F(1,39,50,08)=22,61, p<0,0001$, $\eta_{g}^{2}=0,18$, mittlerer Effekt) (• Abb. 1). Post-hoc-t-Tests für abhängige Stichproben zeigten, dass die Schmerzintensität zwischen dem Messzeitpunkt der Aufnahme $(M=66,5, S D=20,8)$ und der Entlassung $(M=48,2, S D=16,4)(\mathrm{t}(36)=8,5$, $p<0,0001, \mathrm{~d}=1,39$, großer Effekt) sowie zwischen der Aufnahme und drei Monate nach Entlassung $(M=43,2, S D=25,7)$ $(\mathrm{t}(36)=5,3, p<0,0001, \mathrm{~d}=0,88$, großer Effekt) signifikant sank. Es gab keinen signifikanten Unterschied zwischen Entlassung und drei Monate nach Entlassung $(\mathrm{t}(36)=1,2, p=0,68)$.

Analog zur Schmerzintensität zeigte eine weitere einfache ANOVA mit Messwiederholungen einen signifikanten Haupteffekt von Messzeitpunkt auf die schmerzbedingte Beeinträchtigung $(F(1,24,49,64)=36,43, p<0,0001$, $\eta^{2}{ }_{g}=0,25$; mittlerer Effekt) (• Abb. 2). Post-hoc-t-Tests für abhängige Stichproben zeigten, dass die Beeinträchtigung zwischen dem Messzeitpunkt der Auf- 


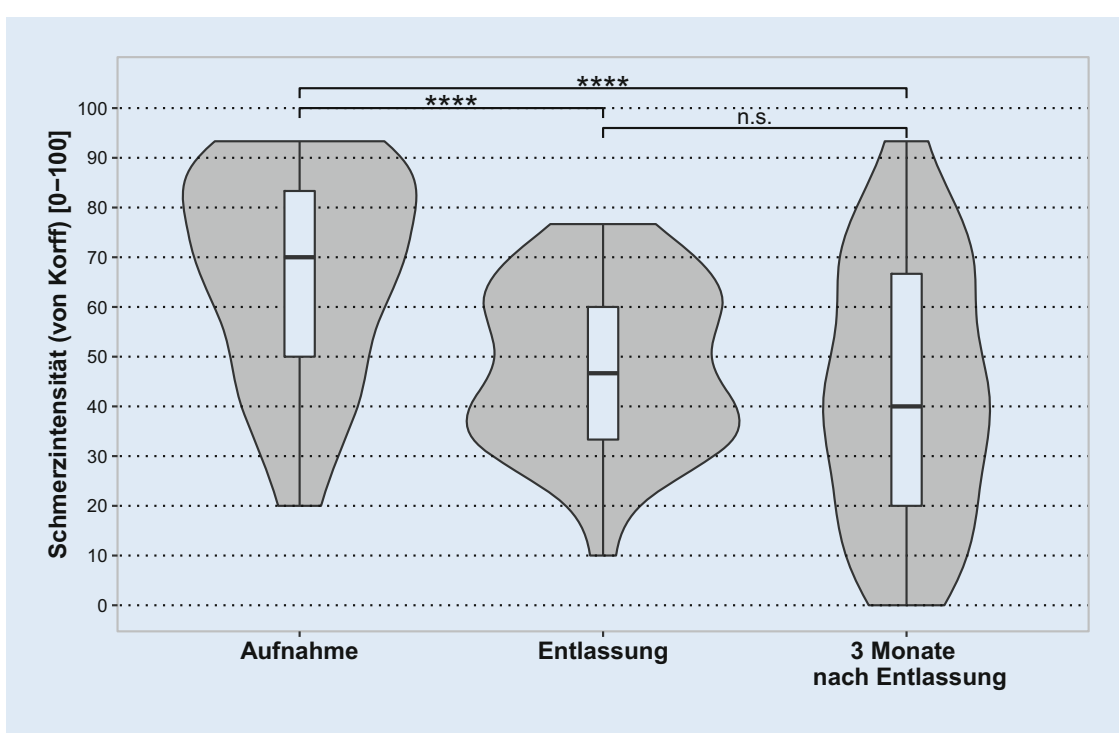

Abb. $1 \Delta$ Schmerzintensität per Messzeitpunkt. ${ }^{* * *} p<0,0001$

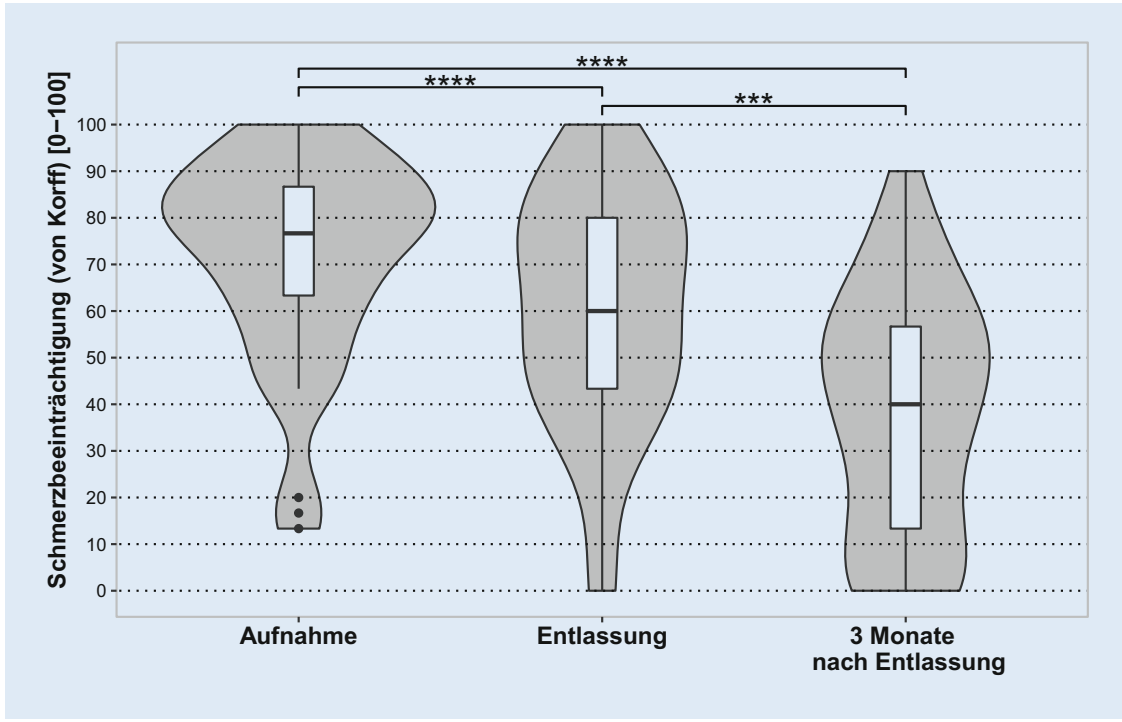

Abb. 2 \ Schmerzbeeinträchtigung per Messzeitpunkt. ${ }^{* * *} p<0,001,{ }^{* * * *} p<0,0001$

nahme $(M=71,5, S D=22,1)$ und der Entlassung $(\mathrm{M}=60,2, \mathrm{SD}=25,2)(\mathrm{t}(40)=6,0$, $p<0,0001, \mathrm{~d}=0,94$, großer Effekt), zwischen Entlassung $(M=60,2, S D=25,2)$ und drei Monate nach Entlassung $(M=38,0$, $\mathrm{SD}=25,9) \quad(\mathrm{t}(40)=4,6, p<0,001, \mathrm{~d}=0,71$, mittlerer Effekt) sowie zwischen der Aufnahme $(M=71,5, S D=22,1)$ und drei Monate nach Entlassung $(M=38,0, S D=25,9)$ $(\mathrm{t}(40)=7,4, p<0,0001, \mathrm{~d}=1,15$, großer Effekt) signifikant sank.
Zusammenhang zwischen Therapieerwartung und Schmerzlinderung

Die Regressionsanalyse zeigte einen signifikanten Zusammenhang zwischen der Therapieerwartung vor Beginn der Behandlung und der Schmerzlinderung über die drei Messzeitpunkte $(\beta=1,98$, $\mathrm{t}(35)=2,4, p<0,05$ ) (siehe - Tab. 3). Die individuelle Therapieerwartung, erhoben mit der Erwartungsskala des CEQ, erklärte $14,6 \%$ der Varianz in Schmerzlinderung $\left(\mathrm{R}^{2}=0,15, \mathrm{~F}(1,35)=6,00, p<0,05, \mathrm{f}^{2}=0,18\right.$, mittlerer Effekt) (• Abb. 3). Es zeigte sich kein signifikanter Zusammenhang zwi- schen der Therapieerwartung und der Beeinträchtigungslinderung.

\section{Diskussion}

In diesem Artikel diskutierten wir kurz die bestehende Literatur zu Erwartungseffekten bei der Behandlung von Schmerzerkrankungen und erweiterten diese durch exemplarische Daten der IMST unseres Hauses. Dies stellt einen ersten Nachweis dieser Einflussgröße in der besonderen schmerztherapeutischen Strukturlandschaft in Deutschland dar.

\section{Effektivität der IMST}

In Übereinstimmung mit vorherigen Studien deuten auch unsere Daten auf die Effektivität der IMST für chronischen Rückenschmerzpatienten hin. Die hier gemessenen Effektstärken übersteigen die eher kleinen Effektstärken, die international beschrieben wurden [29]. Möglicherweise begründet sich dies in den vergleichsweise günstigen Rahmenbedingungen der IMST in Deutschland. Die hier erarbeiteten Konzepte zu Struktur-, Prozess- und Ergebnisparametern fehlen im internationalen Raum noch, was die dortige Standardisierung und Evaluation erschwert [53]. Diese Annahme wird durch ähnliche Ergebnisse vergleichbarer deutscher Beobachtungsstudien gestützt $[9,43,54]$.

\section{Erwartung als Prädiktor für Schmerzlinderung}

Der Einfluss von Erwartung auf die Behandlung von Schmerzen ist bekannt bei Medikamenten [8], Akupunktur [70], kognitiver Verhaltenstherapie [22] sowie multimodaler Schmerztherapie $[14,63]$ und deckt sich somit mit unseren Ergebnissen. Die hier beobachtete mittlere Effektstärke in Verbindung mit 15\% erklärter Varianz verdeutlicht, dass die Erwartungshaltung des Patienten einen nicht zu vernachlässigenden Teil des Behandlungserfolgs ausmacht. Da es sich jedoch bei der Pilotstudie um eine Beobachtungsstudie handelt, sind keine Aussagen über die Kausalität dieses Zusammenhangs möglich. Auch können wir nicht erschließen, wie die Varianz in 
Tab. 3 Regressionsanalyse

\begin{tabular}{|l|l|l|l|l|}
\hline & \multicolumn{2}{|l|}{ Einfluss auf Schmerzlinderung } & \multicolumn{2}{l|}{ Einfluss auf Beeinträchtigungslinderung } \\
\hline & Koeffizient & SD & Koeffizient & SD \\
\hline Konstante & $10,72^{* * *}$ & - & $4,47^{* * *}$ & - \\
\hline Erwartung & $1,98^{*}$ & 0,81 & 0,12 (n.s.) & 0,10 \\
\hline$R^{2}$ & 0,15 & - & 0,04 & - \\
\hline Korr. $R^{2}$ & 0,12 & - & 0,01 & - \\
\hline$F(1,35) /(1,39)$ & $6,00^{*}$ & - & 1,56 (n.s.) & - \\
\hline${ }^{*} p<0,05,{ }^{* *} p<0,001$ & \multicolumn{5}{l}{} \\
\hline
\end{tabular}

der Erwartung unserer Patienten zustande kommt.

Erwartungen können u.a. durch Vorerfahrungen, Konditionierungsprozesse, verbale Suggestion und soziale Lernprozesse, sowohl explizit als auch implizit, entstehen [7, 13]. Es liegt nahe, dass interindividuelle Unterschiede in Patientendispositionen und Vorerfahrungen zu divergenten Erwartungsprofilen und damit zu variierenden Ergebnissen führen. Bräscher et al. (2018) vermuten, dass konditionierungsinduzierte Erwartungseffekte eine große Rolle bei chronischen Schmerzpatienten spielen [10]. Eigenschaften von Schmerzpatienten, wie defizitäre Extinktionsfähigkeit oder eine Historie fehlgeschlagener Behandlungsversuche, könnten Erwartungseffekte zum negativen Pol verschieben. Auch morphologische Unterschiede im Kortex von chronischen Schmerzpatienten [40] könnten die erwartungsinduzierte deszendierende Schmerzhemmung stören. Zudem zeigen unterschiedliche Subgruppen von Schmerzpatienten variierende psychosoziale Profile [11, 18, 64], welche wiederum divergente Erwartungstendenzen implizieren. So können ungünstige Überzeugungen eine Chronifizierung fördern [66] und, wenn sie nicht adressiert werden, die Behandlung stören [69].

Um den Einfluss von Erwartungen therapeutisch zu nutzen, wurden bereits erwartungsmodulierende Interventionen entwickelt. So haben z. B. Rief und Glombiewski (2016) einen erwartungsfokussierten Therapieansatz für die psychotherapeutische Praxis entwickelt [55]. Hierdurch können positive Erwartungen induziert und gefördert werden. In diesem Rahmen sind psychoedukative Gruppen, Erfolgsberichte von vorherigen Patienten (soziales Lernen) oder hypnotherapeutische Suggestionen denkbar. Andererseits könnten pathogene oder generalisierte negative Erwartungen expliziert und adressiert werden. Hierfür wären Methoden der kognitiven Umstrukturierung, aber auch Reizexpositionen denkbar [55]. Durch derartige Interventionen ließen sich bereits Erfolge bei chronischen Rückenschmerzen erzielen [32, 35, 37, 59, 71]. Zukünftige kontrollierte Interventionsstudien sollten untersuchen, welche Erwartungsmodulationen am effektivsten sind und welche interindividuellen Patientenvariablen hierbei berücksichtigt werden sollten. Für eine Übersicht möglicher Strategien zur Optimierung von Erwartungseffekten siehe auch Bingel et al. (2020) [7]. Die Modulierbarkeit der Erwartungen legt nahe, solche Interventionen zu validieren und ihr therapeutisches Potenzial gezielt zu nutzen.

\section{Erwartung hat keinen Einfluss auf Beeinträchtigung}

Der fehlende Nachweis eines Zusammenhangs zwischen der Besserung der Beeinträchtigung und der Erwartung widerspricht scheinbar den Ergebnissen anderer Studien [14, 63]. Unsere Patienten schienen hinsichtlich der Beeinträchtigung, unabhängig von spezifischen Erwartungen, zu profitieren. Eine mögliche Erklärung ist die Beschaffenheit des CEQ, dessen Items das Ausmaß der erwarteten Linderung ermitteln. Möglichweise beziehen Patienten diese Formulierung vorrangig quantitativ auf die Schmerzintensität. Der Fragebogen wäre insofern nicht geeignet, allgemeine oder beeinträchtigungsbezogene Therapieerwartungen zu erfassen. Kube et al. (2017) weisen darauf hin, dass Erwartungen keinen allgemeinen additiven Effekt auslösen, sondern eher spezifische Erwartungen mit den eigentlichen Wirkfaktoren einer Behandlung interagieren [33]. Für zu- künftige Studien empfehlen wir deshalb die Verwendung von Fragebögen, die unterschiedliche Erwartungen und Vorerfahrungen bzgl. unterschiedlicher Therapieelemente und -effekte getrennt voneinander erfassen. Ein Beispiel hierfür wäre die Generische Rating Scale für vorherige Behandlungserfahrungen, Erwartungen und Effekte (GEEE) [56].

\section{Limitationen}

Bei dieser Pilotstudie handelt es sich um eine prospektive longitudinale Beobachtungsstudie. Da die Daten im klinischen Alltag erhoben und genutzt wurden, sind sie weder randomisiert noch kontrolliert. Angesichts der gewöhnlichen Persistenz von chronischen Rückenschmerzen [60] erscheint es jedoch unwahrscheinlich, dass die hier gefundenen Effekte lediglich den spontanen Krankheitsverlauf beschreiben. Die Verwendung des von-Korff-Fragebogens ist aus folgenden Gründen kritisch zu sehen: Die Schmerzintensität-Skala erfasst den Zeitraum der letzten vier Wochen, die Skala der Beeinträchtigung den Zeitraum der letzten drei Monate. Beide Zeiträume übersteigen somit den Zeitraum der Therapie. Dass die Ergebnisse dennoch deutliche Besserungen nach drei bzw. zwei Wochen Therapie zeigen, ist möglicherweise die Folge des subjektiven Eindrucks der Patienten, welcher sich nicht zwangsläufig an objektivierbaren Zeitabschnitten orientierte. Wir möchten zudem darauf hinweisen, dass die Besserungen bis drei Monate nach Therapieende zumindest persistierten und teilweise weiter zunahmen.

Darüber hinaus schien die geistige Gesundheit in unserer Stichprobe geringer zu sein als die von anderen Schmerzpatienten. Dies muss jedoch nicht bedeuten, dass die gefundenen Erwartungseffekte nur oder besonders bei Patienten mit reduzierter geistiger Gesundheit wirken. Da ähnliche Erwartungseffekte bei einer Vielzahl von somatischen Beschwerden bekannt sind, ist anzunehmen, dass es sich hierbei nicht vordergründig um subgruppenspezifische, sondern um allgemeingültige Phänomene handelt. Jedoch gibt es durchaus Hinweise, dass gewisse psychologische Eigenschaften und psychische Zustände die modulierende Wirkung von Erwartungen beeinflussen können [7]. 


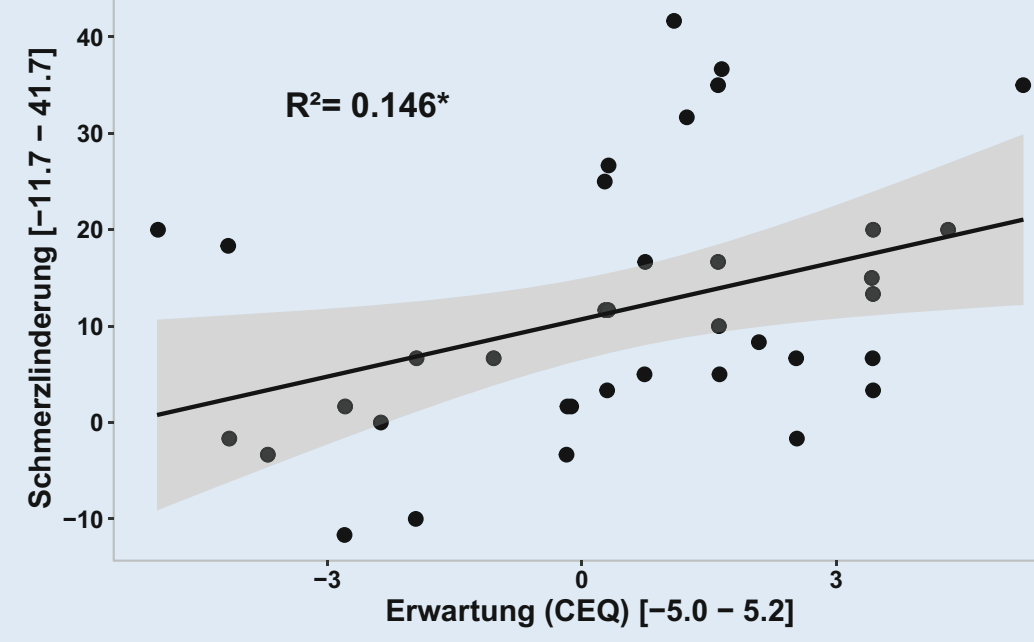

Abb. $3 \Delta$ Assoziation der Therapieerwartung und der Schmerzlinderung drei Monate nach IMST. ${ }^{*} p<0,05$

So können Anspannung, Stress und negativer Affekt die Wirkung von positiver Erwartung hemmen und die von negativer Erwartung verstärken. Auch mehr persistierende Zustände wie Ängste und Depressivität zeigen ähnliche Auswirkungen [34, 58]. Wenn reduzierte geistige Gesundheit einen ähnlich hemmenden Einfluss wie Depressivität hat, dann könnte es sein, dass der Effekt von Erwartung auf das Ergebnis durch unsere Ergebnisse noch unterschätzt wird. Zukünftige Studien sollten die Übertragbarkeit unserer Ergebnisse auch auf andere Patientenkollektive und die mediierende Wirkung von positiver geistiger Gesundheit überprüfen.

Des Weiteren sei zu erwähnen, dass wir keine Aussage über den Verlauf und den Einfluss von Erwartung der Patienten treffen können, die nicht alle Messzeitpunkte eingehalten haben. Zumindest scheinen sie sich zum Einschlusszeitpunkt nicht signifikant hinsichtlich der primären Endpunkte unterschieden zu haben. Dies ist eine generelle Limitation klinischer Studien.

\section{Ausblick und Fazit für die Praxis}

Die bereits metaanalytisch belegte, klinisch relevante Effektivität der IMST spiegelt sich auch in unserer untersuchten Kohorte wider. Unsere Pilotuntersuchung bestätigt einen bereits in anderen experimentellen und klinischen Kontexten doku- mentierten Zusammenhang zwischen der Therapieerwartung und dem Therapieergebnis. Auch wenn unsere Untersuchung aus methodischen Gründen keinen kausalen Zusammenhang der genannten Faktoren herstellen kann, unterstützt unsere Beobachtung in Zusammenschau mit experimentellen und kontrollierten klinischen Untersuchungen die Bedeutung der Therapieerwartung als eine wichtige Einflussgröße des Behandlungserfolgs. Obwohl diese Einflussgröße bereits in einer Vielzahl von experimentellen Studien beschrieben und untersucht wurde, wird sie aktuell in der klinischen Praxis kaum systematisch genutzt. Die hier vorgestellte Untersuchung soll dazu motivieren, die Bedeutung der Erwartung im schmerztherapeutischen Kontext inklusive der IMST in größeren Kohorten besser und systematischer zu untersuchen. Hierbei sind sowohl die Mechanismen der Erwartungsbildung als auch deren Effekte auf den Therapieerfolg von Bedeutung. Eine weitere wesentliche Herausforderung ist das Verständnis von interindividuellen Unterschieden der Erwartung und von Erwartungseffekten. Ein besseres Verständnis dieser Einflussgrößen sowie von dynamischen Veränderungen der Erwartung im longitudinalen Verlauf von Behandlungen sind die Grundlage, um die individuelle Therapieerwartung gezielt und zum Wohle des Patienten zu modulieren. Solche gezielten Interventionen könnten ein fester Bestandteil in der
Schmerztherapie, ganz besonders im Rahmen der "kognitiv-behavioralen“Elemente der IMST, sein. In einer weiterführenden Studie planen wir, den Einfluss einer erwartungsmodulierenden Intervention auf den Effekt der IMST zu untersuchen. Hierbei wird eine Gruppe, welche die erwartungsmodulierende Intervention erhält, mit der gewohnten IMST-Gruppe verglichen. Zusätzlich planen wir hierbei die laufende Warteliste der IMST als Kontrollgruppe zu nutzen.

\section{Korrespondenzadresse}

\section{Dustin Maser}

Klinik für Neurologie, Zentrum für Translationale Neuro- und Verhaltenswissenschaften und Zentrum für universitäre Schmerzmedizin, Universitätsklinikum Essen (AöR) Hufelandstraße 55, 45147 Essen, Deutschland dustin.maser@uk-essen.de

Förderung. Gefördert durch die Deutsche Forschungsgemeinschaft (DFG) - Projektnummer 422744262-TRR 289

Funding. Open Access funding enabled and organized by Projekt DEAL.

\section{Einhaltung ethischer Richtlinien}

Interessenkonflikt. D. Maser, D. Müller, U. Bingel und D. Müßgens geben an, dass kein Interessenkonflikt besteht. Alle Co-Autoren haben das vorliegende Manuskript gelesen und stimmen den Inhalten zu. In diesem Kontext haben die Autoren kein finanzielles Interesse anzugeben.

Die vorliegende Pilotstudie ist Teil einer größeren retrospektiven und prospektiven Studie über die interin dividuellen Unterschiede und psychologischen Prädiktoren der interdisziplinären multimodalen Schmerztherapie am Universitätsklinikum Essen (Ethikvotum: 18-8251-BO). Die Studie ist registriert im Deutschen Register Klinischer Studien unter DRKS00022951.

Open Access. Dieser Artikel wird unter der Creative Commons Namensnennung 4.0 International Lizenz veröffentlicht, welche die Nutzung, Vervielfältigung, Bearbeitung, Verbreitung und Wiedergabe in jeglichem Medium und Format erlaubt, sofern Sie den/die ursprünglichen Autor(en) und die Quelle ordnungsgemäß nennen, einen Link zur Creative Commons Lizenz beifügen und angeben, ob Änderungen vorgenommen wurden.

Die in diesem Artikel enthaltenen Bilder und sonstiges Drittmaterial unterliegen ebenfalls der genannten Creative Commons Lizenz, sofern sich aus der Abbildungslegende nichts anderes ergibt. Sofern das betreffende Material nicht unter der genannten Creative Commons Lizenz steht und die betreffende Handlung nicht nach gesetzlichen Vorschriften erlaubt ist, ist für die oben aufgeführten Weiterverwendungen des Ma- 


\section{Originalien}

terials die Einwilligung des jeweiligen Rechteinhabers einzuholen.

Weitere Details zur Lizenz entnehmen Sie bitte der Lizenzinformation auf http://creativecommons.org/ licenses/by/4.0/deed.de.

\section{Literatur}

1. Arnold B, Brinkschmidt T, Casser HR et al (2014) Multimodale Schmerztherapie für die Behandlung chronischer Schmerzsyndrome. Schmerz 28:459-472

2. Arnold B, Brinkschmidt T, Casser HR et al (2009) Multimodale Schmerztherapie. Schmerz 23:112

3. Ashar YK, Chang LJ, Wager TD (2017) Brain mechanisms of the placebo effect: an affective appraisal account. Annu Rev Clin Psychol 13:73-98

4. Auer CJ, Glombiewski JA, Doering BK et al (2016) Patients' expectations predict surgery outcomes: a meta-analysis. IntJ Behav Med 23:49-62

5. Bfarm (2020) ICD-10-GM Version 2020, Systematisches Verzeichnis, Internationale statistische Klassifikation der Krankheiten und verwandter Gesundheitsprobleme, 10. Revision, Stand: 18. September 2020. Bundesinstitut für Arzneimittel und Medizinpodukte (BfArM), Köln

6. Bfarm (2020) OPS Version 2021, Systematisches Verzeichnis, Operationen- und Prozedurenschlüssel Internationale Klassifikation der Prozeduren in der Medizin (OPS), Stand:08. Dezember 2020. Bun desinstitut für Arzneimittel und Medizinprodukte (BfArM), Köln

7. Bingel U (2020) Placebo 2.0: the impact of expectations on analgesic treatment outcome. Pain 161(1):S48-s56

8. Bingel U, Wanigasekera V, Wiech K et al (2011) The effect of treatment expectation on drug efficacy: imaging the analgesic benefit of the opioid remifentanil. Sci Transl Med 3:70ra14

9. Borys C, Lutz J, Strauss B et al (2015) Effectiveness of a multimodal therapy for patients with chronic low back pain regarding pre-admission healthcare utilization. PLoS ONE 10:e143139

10. Bräscher AK, Witthöft M, Becker S (2018) The underestimated significance of conditioning in placebo hypoalgesia and Nocebo hyperalgesia. Pain Res Manag 2018:6841985

11. Buchmann J, Baumann N, Meng K et al (2021) Endurance and avoidance response patterns in pain patients: application of action control theory in pain research. PLoSONE 16:e248875-e248875

12. Casser HR, Arnold B, Gralow I et al (2013) Interdisziplinäres Assessment zur multimodalen Schmerztherapie. Schmerz 27:363-370

13. Colloca L, Miller FG (2011) How placebo responses are formed: a learning perspective. Philos Trans $\mathrm{R}$ Soc Lond B Biol Sci 366:1859-1869

14. Cormier $S$, Lavigne $G L$, Choinière $M$ et al (2016) Expectations predict chronic pain treatment outcomes. Pain 157:329-338

15. Devilly GJ, Borkovec TD (2000) Psychometric properties of the credibility/expectancy questionnaire. J Behav Ther Exp Psychiatry 31:73-86

16. Dragioti E, Evangelou E, Larsson B et al (2018) Effectiveness of multidisciplinary programmes for clinical pain conditions: an umbrella review. JRehabil Med 50:779-791

17. Dutmer AL, Schiphorst Preuper HR, Soer $R$ et al (2019) Personal and societal impact of low back pain: the Groningen spine cohort. Spine 44(24):E1443-E1451. https://doi.org/10.1097/ BRS.0000000000003174
18. Fillingim RB (2017) Individual differences in pain: understanding the mosaic that makes pain personal. Pain 158(1):S11-s18

19. Flaten MA, Simonsen T, Olsen H (1999) Drug related information generates placebo and nocebo responses that modify the drug response. Psychosom Med 61:250-255

20. GaulC, van DoornC, Webering Netal (2011)Clinica outcome of a headache-specific multidisciplinary treatment program and adherence to treatment recommendations in a tertiary headachecenter:an observational study. J Headache Pain 12:475-483

21. Göbel H (2001) Epidemiologie und Kosten chronischer Schmerzen. Schmerz 15:92-98

22. Goossens ME, Vlaeyen JW, Hidding A et al (2005) Treatment expectancy affects the outcome of cognitive-behavioral interventions in chronic pain. Clin JPain 21:18-26 (discussion 69-72)

23. Häuser W, Bernardy K, Arnold B et al (2009) Efficacy of multicomponent treatment in fibromyalgia syndrome: a meta-analysis of randomized controlled clinical trials. Arthritis Rheum 61:216-224

24. Hayden JA, Wilson MN, Riley RD et al (2019) Individual recovery expectations and prognosis of outcomes in non-specific low back pain: prognostic factor review. Cochrane Database Syst Rev 11:CD11284

25. Herda C, Scharfenstein A, Basler H-D (1998) Marburger Fragebogen zum habituellen Wohlbefinden. Schriftenreihe des Zentrums Methodenwissenschaften und Gesundheitsforschung. Marburg:Philipps-Universität, Marburg (Arbeitspapier 98-1)

26. Hoy D, March L, Brooks P et al (2014) The global burden of low back pain: estimates from the globa burden of disease 2010 study. Ann Rheum Dis 73:968-974

27. Kaiser U, Sabatowski R, Azad CS (2015) Multimodal pain therapy : current situation. Schmerz 29(5):550-556. https://doi.org/10.1007/s00482015-0030-4

28. Kaiser U, Treede R, Sabatowski R (2017) Multimodal pain therapy in chronic noncancer pain-gold standard or need for further clarification? Pain 158:1853-1859

29. Kamper SJ, Apeldoorn AT, Chiarotto A et al (2014) Multidisciplinary biopsychosocial rehabilitation for chronic low back pain. Cochrane Database Syst Rev 9:CD963

30. Kazis LE, Selim A, Rogers W et al (2006) Dissemination of methods and results from the veterans health study: final comments and implications fo future monitoring strategies within and outside the veterans healthcare system. J Ambul Care Manag 29:310-319

31. Koyama T, Mchaffie JG, Laurienti PJ et al (2005) The subjective experience of pain: where expectations become reality. Proc Natl Acad Sci USA 102:12950-12955

32. Kube T, Glombiewski JA, Rief W (2018) Using different expectation mechanisms to optimize treatment of patients with medical conditions: a systematic review. Psychosom Med 80:535-543

33. Kube T, RiefW (2017) Are placeboand drug-specific effects additive? Questioning basic assumptions of double-blinded randomized clinical trials and presenting novel study designs. Drug Discov Today 22:729-735

34. Lee HF, Hsieh JC, Lu CLet al (2012) Enhanced affect/ cognition-related brain responses during visceral placebo analgesia in irritable bowel syndrome patients. Pain 153:1301-1310

35. Leeuw M, Goossens M, van Breukelen GJP et al (2008) Exposure in vivo versus operant graded activity in chronic low back pain patients: results of a randomized controlled trial. Pain 138:192-207

36. Liccardi G, Senna G, Russo Met al (2004) Evaluation of the nocebo effect during oral challenge in patients with adverse drug reactions. J Investig Allergol Clin Immunol 14:104-107

37. Linton SJ, Boersma K, Jansson M et al (2008) A randomized controlled trial of exposure in vivo for patients with spinal pain reporting fear of workrelated activities. Eur JPain 12:722-730

38. Lombardi C, Gargioni S, Canonica GW et al (2008) The nocebo effect during oral challenge in subjects with adverse drug reactions. Eur Ann Allergy Clin Immunol 40:138-141

39. Maher C, Underwood M, Buchbinder R (2017) Nonspecific low back pain. Lancet 389:736-747

40. May A (2008) Chronic pain may change the structure of the brain. Pain 137:7-15

41. Mcrae C, CherinE, Yamazaki TGetal (2004) Effects of perceived treatment on quality of life and medical outcomes in a double-blind placebo surgery trial. Arch Gen Psychiatry 61:412-420

42. Meints SM, Edwards RR (2018) Evaluating psychosocial contributions to chronic pain outcomes. Prog Neuropsychopharmacol Biol Psychiatry 87:168-182

43. Moradi B, Hagmann S, Zahlten-Hinguranage A et al (2012) Efficacy of multidisciplinary treatment for patients with chronic low back pain: a prospective clinical study in 395 patients. J Clin Rheumatol 18:76-82

44. Moseley JB, O'Malley K, Petersen NJ et al (2002) A controlled trial of arthroscopic surgery for osteoarthritis of the knee. NEngl J Med 347:81-88

45. Moser U (2019) Interdisziplinäre multimodale Schmerztherapie - quo vadis? Schmerz 33:555-557

46. Murgatroyd DF, Harris IA, Tran Y et al (2016) Predictors of return to work following motor vehicle related orthopaedic trauma. BMC Musculoskelet Disord 17:171

47. Myers MG, Cairns JA, Singer J (1987) The consent form as a possible cause of side effects. Int J Clin Pharmacol Ther 42:250-253

48. Nilges P, Essau C (2015) Die Depressions-AngstStress-Skalen. Schmerz 29:649-657

49. Peerdeman KJ, van Laarhoven AIM, Keij SM et al (2016) Relieving patients' pain with expectation interventions: a meta-analysis. Pain 157:1179-1191

50. Petzke F, Hüppe M, Kohlmann T et al (2020) Handbuch Deutscher Schmerz-Fragebogen. Deutsche Schmerzgesellschaft e.V. https:// www.schmerzgesellschaft.de/fileadmin/pdf/ DSF_Handbuch_2020.pdf. Zugegriffen: 06. August 2021

51. Pfingsten M, Arnold B, Böger A et al (2019) Sektorenübergreifende interdisziplinäre multimodale SchmerztherapieCross-sectoral interdisciplinary multimodal pain therapy: Empfehlungen zu Struktur- und Prozessparametern der Adhoc-Kommission „Interdisziplinäre Multimodale Schmerztherapie" der Deutschen Schmerzgesellschaft e. V.Recommendations on structural and process parameters of the ad hoc commission "Interdisciplinary Multimodal Pain Therapy" of the German Pain Society (Deutsche Schmerzgesellschaft e. V.). Schmerz. https://doi.org/10.1007/ s00482-019-0374-2

52. Pfingsten M, Arnold B, Böger A et al (2019) Crosssectoral interdisciplinary multimodal pain therapy : recommendations on structural and process parameters of the ad hoc commission "interdisciplinary Multimodal pain therapy" of the German 
pain society (Deutsche Schmerzgesellschaft e. V.). Schmerz 33:191-203

53. Pfingsten M, Kaiser U, Sabatowski R (2019) Qualität und Effektivität der interdisziplinären multimodalen Schmerztherapie. Schmerz 33:558-561

54. Pöhlmann K, Tonhauser T, Joraschky P et al (2009) The Dachau multidisciplinary treatment program for chronic pain. Efficacy data of a diagnosisindependent multidisciplinary treatment program for back pain and other types of chronic pain. Schmerz 23:40-46

55. Rief W, Glombiewski JA (2016) Erwartungsfokussierte Psychotherapeutische Interventionen (EFPI). Verhaltenstherapie 26:47-54

56. Rief W, Nestoriuc Y, Mueller E et al (2021) Generic rating scale for previous treatment experiences, treatment expectations, and treatment effects (GEEE)

57. Scascighini L, Toma V, Dober-Spielmann S et al (2008) Multidisciplinary treatment for chronic pain: a systematic review of interventions and outcomes. Rheumatology 47:670-678

58. Schmid J, Bingel U, Ritter C et al (2015) Neural underpinnings of nocebo hyperalgesia in visceral pain: afMRI study in healthy volunteers. Neuroimage 120:114-122

59. Siemonsma PC, Stuive I, Roorda LD et al (2013) Cognitive treatment of illness perceptions in patients with chronic low back pain: a randomized controlled trial. Phys Ther 93:435-448

60. Smith BH, Elliott AM, Hannaford PC et al (2004) Factors related to the onset and persistence of chronic back pain in the community: results from a general population follow-up study. Spine (Phila Pa 1976) 29:1032-1040

61. Tabachnick BG, Fidell LS (2007) Using multivariate statistics, 5. Aufl. Allyn \& Bacon/Pearson Education, Boston, MA

62. Treede R-D, Rief W, Barke A et al (2015) A classification of chronic pain for ICD-11. Pain 156:1003-1007

63. Tseli E, Vixner L, Lomartire Ret al (2020) Prognostic factors for improved physical and emotional functioning one year after interdisciplinary rehabilitation in patients with chronic pain: results fromanational quality registryin Sweden.J Rehabil Med 52:jrm19

64. Turk DC (2005) The potential of treatment matching for subgroups of patients with chronic pain: lumping versus splitting. Clin J Pain 21(1):44-55

65. Varelmann D, Pancaro C, Cappiello EC et al (2010) Nocebo-induced hyperalgesia during local anesthetic injection. Anesth Analg 110:868-870

66. Vlaeyen JWS, Linton SJ (2000) Fear-avoidance and its consequences in chronic musculoskeletal pain: a state of the art. Pain 85:317-332

67. Vlaeyen JWS, Maher CG, Wiech K et al (2018) Low back pain. Nat Rev Dis Primers 4:52

68. Von Korff M, Ormel J, Keefe FJ et al (1992) Grading the severity of chronic pain. Pain 50:133-149

69. Wertli MM, Rasmussen-Barr E, Held U et al (2014) Fear-avoidance beliefs-a moderator of treatment efficacy in patients with low back pain: a systematic review. Spine J 14:2658-2678

70. Witt CM, Schützler L, Lüdtke R et al (2011) Patient characteristics and variation in treatment outcomes: which patients benefit most from acupuncture for chronic pain? Clin J Pain 27:550-555

71. Woods MP, Asmundson GJG (2008) Evaluating the efficacy of graded in vivo exposure for the treatment of fear in patients with chronic back pain: a randomized controlled clinical trial. Pain 136:271-280

\section{Results of a pilot study on the role of therapy expectation in interdisciplinary multimodal pain therapy for chronic back pain}

Background: Chronic low back pain is a serious persistent illness with profound personal and socioeconomic impact. Interdisciplinary multimodal pain therapy (IMPT) is one of the few evidence-based treatment options for chronic pain. Although it is known that pain perception, as well as its chronification and treatment are affected by patient expectations, only few clinical interventions or guidelines on how to modulate these effects exist.

Objectives: The aim of this study was to demonstrate the impact of expectancy as a predictor for pain and related outcomes. To this end, we will present explorative pilot data from an observational cohort at our clinic.

Methods: The study shows preliminary data of a prospective longitudinal observational study of up to 41 chronic back pain patients who followed an IMPT at the back pain center in Essen. Data were collected at admission (T0), at discharge (T1), and 3 months after discharge (T2). Primary outcomes were pain intensity and disability. Additionally, we measured treatment expectancy at admission. We used linear regression to analyze the impact of pretreatment expectancy on the primary outcome measures.

Results: IMPT led to a significant improvement in pain intensity and disability. The effect on pain intensity was stable over three months after discharge and disability declined even further. Expectancy was a significant predictor of improvement in pain intensity and explained approximately $15 \%$ of the variance.

Discussion: Expectancy is an important predictor of treatment outcome in IMPT. In clinical practice, valid methods should therefore be established to reduce negative and promote positive expectations.

\section{Keywords}

Chronic back pain - Interdisciplinary multimodal pain therapy (IMPT) - Treatment expectancy Placebo - Longitudinal observational study 